\title{
A SCANNING MICROMETER WITH ON-LINE COMPUTER
}

\author{
ERIK HøG \\ Hamburger Sternwarte, Germany
}

\begin{abstract}
A photoelectric scanning photometer is being tested at the $60 \mathrm{~cm}$-refractor in Hamburg in order to measure visual binaries. The data-acquisition is accomplished by a small on-line computer PDP-8/S which samples data from three photon-counters connected to three narrow slits in the focal plane.

A preliminary investigation of the image profile as it is obtained from the adding of many scans of a star on top of each other shows that about $80 \%$ of the light is concentrated in a Gaussian core with $\sigma=1^{\prime \prime}-2^{\prime \prime}$ whereas the rest goes into non-Gaussian wings. The deviation of the profile from a single Gaussian is most pronounced in good seeing.
\end{abstract}

Photoelectric scanning of small objects is becoming more important since a higher resolution and a better photometric accuracy may be obtained than by conventional photography or photoelectric photometry as shown by Rakos (1965) and Franz (1966). The method is gaining impetus from the recent availability of small computers which may be integrated in the electronic system without undue costs.

An on-line computer secures a data sampling capacity, e.g. 1000 measurements per second, otherwise only achieved by magnetic tape and it gives the opportunity of handling the data so that they may be presented to the observer in convenient analog and digital forms. Furthermore, such a data acquisition system is very flexible, e.g. at our telescope the system is used at the scanning micrometer as well as for measurement of star occultations and in a search for rapid variables, in which also the Crab pulsar has been measured.

\section{The Scanning Micrometer}

The image of the double star is moved in any position angle across three narrow adjacent slits in the focal plane of the telescope. Figure 1 shows the optical-mechanical arrangement of the scanning micrometer. The linear motion of the image across the slits is accomplished by a double mirror consisting of two plane aluminized mirrors which rocks around an axis perpendicular to the optical axis and thereby shifting the optical axis parallel to itself.

A thin steel blade with straight edges wound around a cylinder constitutes the cam. Several cams with different heights are available. The arm with roller and the double mirror are so dimensioned that a cam with $4 \mathrm{~mm}$ height moves the image $1 \mathrm{~mm}$. This decrease of scale should improve the accuracy of the linear motion at the rather small scale of $20^{\prime \prime} / \mathrm{mm}$ of the present telescope. It is also of value for the accuracy that the rocking mirror has a very small moment of inertia and that the motion is accomplished by rotations only. A trigger pulse initiated at each revolution by a slotted disc on the axis of the cam is fed to the computer.

The three accurate slits $15 \mu$ wide and $50 \mu$ apart are engraved on a glassplate mounted on a wheel with 5 positions containing other slits, diaphragms and a cross 


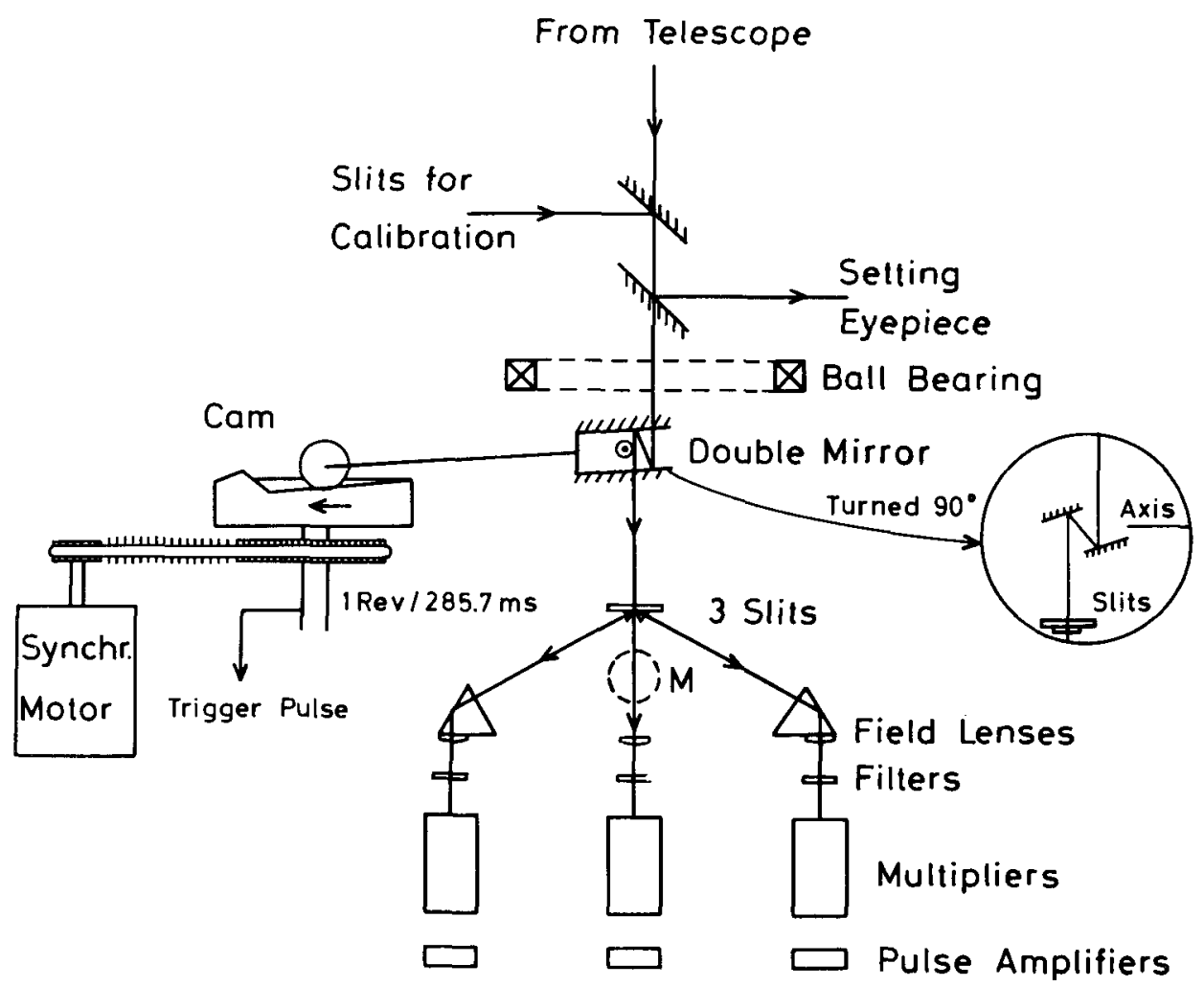

Fig. 1. Scheme of scanning micrometer. The image of the double star is moved in any position angle across three narrow slits each connected with its own photometric channel.

wire. The two outer beams pass through filter wheels accommodating 10 filters whereas the central beam has 3 filters only.

A switching mirror allows an image consisting of two illuminated slits to be observed for purposes of calibration: (1) adjusting the optical system, (2) measuring the linearity of the motion of the image, and (3) photometric checking.

The system is so designed that the image of the telescope objective formed by the field lenses on the photocathodes coincide with the image of the adjustable aperture diaphragm in the calibration beam. Furthermore, the same spots on the cathodes are illuminated when (1) the star image moves across the slits, when (2) the micrometer is turned in position-angle, and when (3) the telescope is pointed at different parts of the sky.

\section{The Data Acquisition System}

Three possibilities exist for sampling 1000 measurements per second: magnetic tape, multichannel-analyzer, on-line computer. We choose the last solution for its greater flexibility in the sampling and in the data handling, and constructed a data acquisition system, Figure 2, suited for a variety of astronomical applications. 


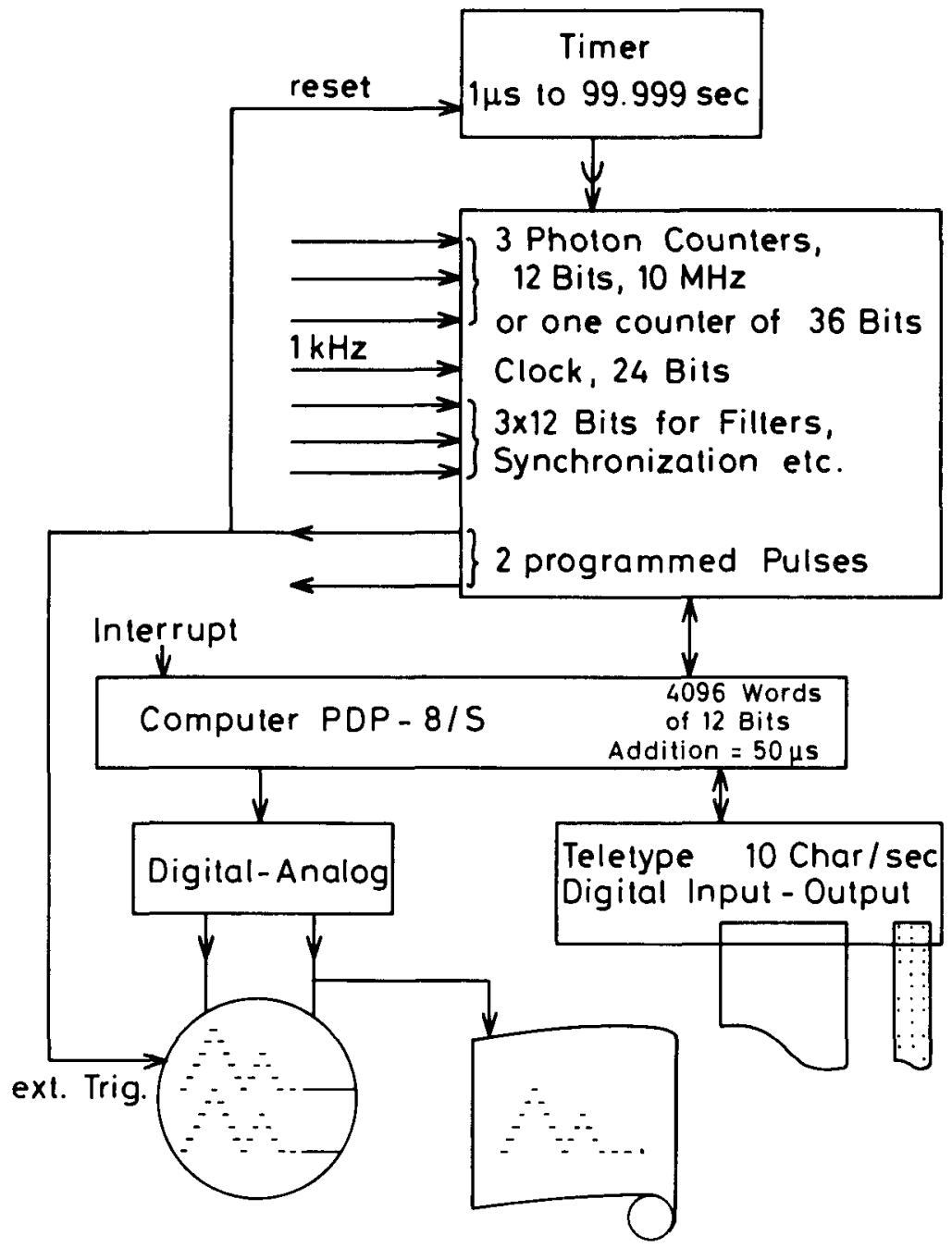

Fig. 2. The data acquisition system with on-line computer is suited for a variety of astronomical applications such as scanning photometry, star occultations, and search for optical pulsars. Up to 1000 measurements per second.

The computer PDP-8/S with a (slow) digital input-output and two digital to analog converters was extended in our electronics workshop with a timer and a number of counters and digital inputs.

The timer initiates at equidistant intervals the transfer of the contents of the three photon counters to their respective storages, resets the counters and sets a status signa] on which the computer may react in the following way. The computer having been waiting in a program loop for the status signal to be set now transfers the contents of the photon counter storages one after another into the computer storage and resets the status signal. A clock counter of 24 bits may be requested or reset by the program and 
36 bits are available for digitalizing filter wheels, synchronizing the clock with Universal Time etc.

In case of the scanning micrometer, the trigger pulse from the cam causes the computer to start sampling at e.g. $4 \mathrm{~ms}$ intervals and to take e.g. 60 values in each photometric channel.

\section{Preliminary Results}

A recording of a double star obtained from the addition of 10 consecutive scans is shown in Figure 3. Obviously, even at this large distance of $7^{\prime \prime}$ the wings of the images

\section{$\Sigma 2276 \quad 7.11 \quad m_{v}=7.0-7.4$ \\ 10 scans with 3 slits}

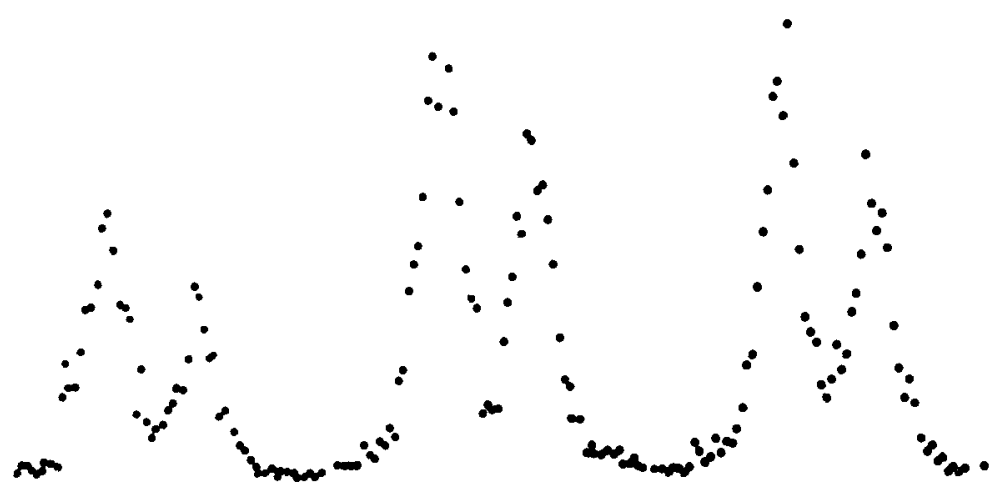

Fig. 3. Recording of a double star with three slits.

overlap. In order to separate the two components by computation it is therefore useful to see which kind of profiles occur in practice.

Image profiles of single stars from different nights are plotted in Figure 4. They differ primarily because of different seeing conditions. The narrow core of the image may be explained by the diffraction disc which is broadened by image motion when adding the consecutive scans on top of each other. The wider wings are formed by light from different parts of the objective which are so much out of phase that they form their own individual diffraction discs. The weighted sum of these larger discs is the main constituent of the wings; see Meinel (1960).

The influence of the assumed mathematical form of the image profile on the measured magnitude difference $\Delta m$ is small if $\Delta m$ is small but serious systematic errors 
may occur in other cases. If Gaussian profiles are fitted to the observations of equal weights they will actually be forced to fit the cores and to predict fainter wings than really are. For a pair of $\varrho=4^{\prime \prime}$ and $\Delta m=2^{m} .5$ and an image profile as No. 3 in Figure 4, $\Delta m$ would be measured about $0^{m} .1$ systematically too small. The best check on systematic errors is to measure a wide pair in different position angles and to make sure that $\Delta m$ does not change and that the projected distance is correctly measured.

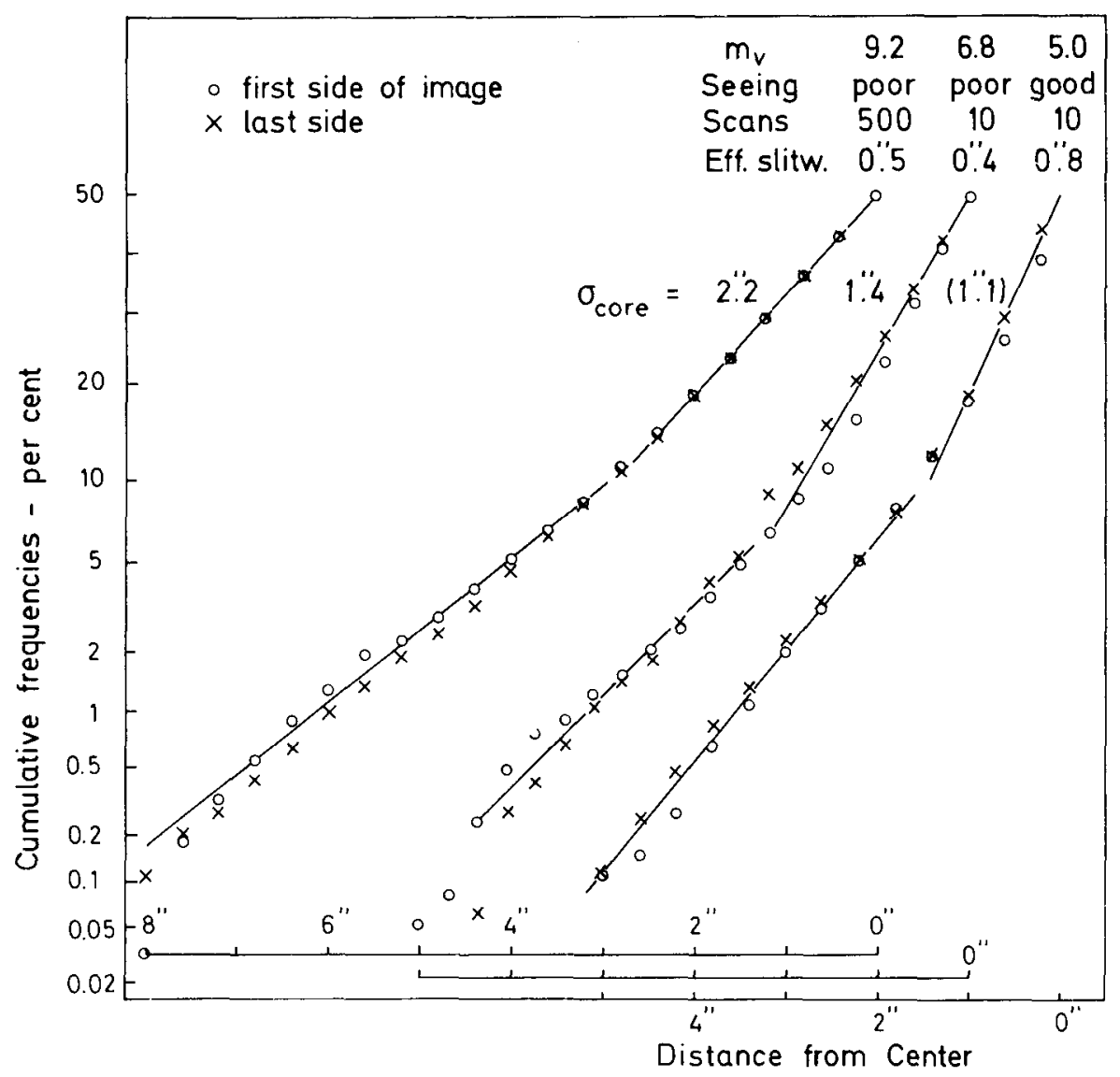

Fig. 4. Image profiles from nights of different quality showing a clear deviation from a simple Gaussian which would be one straight line. Instead it appears that about $80 \%$ of the light is concentrated in a nearly Gaussian core and the rest in wider non-Gaussian wings. And, clearly, the better the seeing is the larger the deviation from a Gaussian.

The possibility of using an image profile measured at a near-by single star for the reduction may have its advantages. But the best solution is probably to assume only that the image profile is symmetric since this allows to use the hardly disturbed outside wings to separate the overlapping inside wings of the double star. 


\section{References}

Franz, O. G.: 1966, Lowell Obs. Bull. No. 134.

Meinel, A. B.: 1960, 'Astronomical Seeing and Observatory Site Selection', in Stars and Stellar Systems, I, Univ. of Chicago Press, 157, Chicago-London.

Rakos, K. D.: 1965, Appl. Opt. 4, 1453.

\section{Discussion}

The infiuence of the profiles is further discussed by Rösch and Franz; theory and the different observations are in essential agreement. Høg tries to show the seeing influence by a model: Imagine the telescope area divided into cells, and images formed from different cell groups. Cells with nearly equal wave-front phases will produce a diffraction-limited profile unaffected by seeing; otherwise the wings will be enhanced without materially altering the dispersion of the quasi-Gaussian core. 